\title{
Massive Primordial Black Holes
}

\author{
A.D. Dolgov ${ }^{* \dagger}$ \\ Novosibirsk State University and ITEP, Russia \\ E-mail: dolgovafe.infn.it
}

A review of the astronomical data of several last years on an astonishingly high amount of black holes in the contemporary and early $(z \sim 10)$ universe is presented. Also the data on the recently observed peculiar stars in the Galaxy are discussed. It is argued that practically all black holes in the universe are primordial $(\mathrm{PBH})$ and suggested that an inverted picture of the galaxy formation is realized: supermassive black holes were formed prior to galaxy formation and subsequently seeded the latter. Possibilities of cosmological dark matter consisting of primordial black holes and of abundant cosmological antimatter are considered.

A mechanism of 1993 anticipating all these phenomena and predicting an extended log-normal mass spectrum of $\mathrm{PBH}$ is described.

Multifrequency Behaviour of High Energy Cosmic Sources - XIII - MULTIF2019

3-8 June 2019

Palermo, Italy

\footnotetext{
* Speaker.

${ }^{\dagger}$ The support of the RNF Grant 19-42-02004 is acknowledged.
} 


\section{Introduction}

Recent astronomical data, which keep on appearing almost every day, show that the contemporary, $z \sim 0$, universe is much more abundantly populated by all kind of black holes, than it was expected even a few years ago. There are lots of black holes in all mass intervals:

-massive, from a fraction of $M_{\odot}$ up to $\gtrsim 100 M_{\odot}$,

-supermassive (SMBH), $M \sim\left(10^{6}-10^{9}\right) M_{\odot}$,

- intermediate mass (IMBH) $M \sim\left(10^{3}-10^{5}\right) M_{\odot}$,

The origin of most of these black hole is not clear, to say the least.

In addition, there is a population of peculiar stars in the Galaxy (too old, too fast, and with an unusual chemical content) which are at odds with the conventional astrophysics.

Moreover, the data collected during last several years indicate that the young universe at $z \sim 10$ is grossly overpopulated with unexpectedly high amount of:

- bright QSOs, alias supermassive BHs, with masses up to $M \sim 10^{10} M_{\odot}$;

- superluminous young galaxies;

- supernovae, gamma-bursters;

- dust and heavy elements.

The canonical mechanism of formation of SMBH even in the present day universe demands at least an order of magnitude longer time than the universe age, $t_{U}=14.6 \cdot 10^{9}$ years, and the problem of their formation in ten times younger universe at $z \sim 10$ is multifold more pronounced.

In this talk a review of these surprising astronomical observations is presented and the mechanism suggested a quarter of century ago of primordial black hole (PBH) and peculiar star formation $[1,2]$, which neatly solves the above mentioned problems, is described. Not only abundant PBHs but also a significant amount of rather strange primordial stars are predicted, They may make a considerable or even $100 \%$ contribution to the cosmological dark matter. To some extent the content of this talk coincides with the author's review [3], but of course during the last one-two years much more new observational data have been obtained.

\section{Black Holes: what's that}

\subsection{Observations of black holes}

Existence of black holes (BHs) was first envisaged by John Mitchell in 1784, who predicted that there might be bodies with so strong gravitational field that the second cosmic velocity would be larger than the speed of light. He concluded that they neither shine not reflect light, i.e. they are absolutely dark, and it is impossible to observe to them.

This is not precisely true, because BHs can emit all kind of radiation in the process of their evaporation predicted by Stephen Hawking and, though nobody yet has seen it, there are no doubts that such process exists. The life-time of a $\mathrm{BH}$ with respect to evaporation scales as its mass cube, and for $M_{B H}=5 \cdot 10^{14} \mathrm{~g}$ the life-time is close to the universe age $t_{U} \approx 10^{10}$ years. So the stellar mass BHs (and of course heavier ones) will survive even after all the protons in the universe will decay, since their life-time is presumably finite, about $10^{33}$ years.

BHs strongly interact with the surrounding matter and it makes them very visible. For example, the most powerful sources of radiation in the universe are quasars (QSO) which are pointl-like 
objects, within the accuracy of the telescopes, and shine as thousands of galaxies. The only plausible mechanism of explanation for the operation of the central QSO engine is the emission of radiation in the process of collisions of ultra-relativistic particles accreting to a supermassive black hole $(\mathrm{SMBH})$ in the center. Such process can heat the surrounding matter up to million degrees.

Less massive $\mathrm{BHs}$ are also observed through emission of X-rays from the heated gas around them.

Another way to observe $\mathrm{BHs}$ is based on studying the star motion around an invisible gravitating object of small size. That's how the central BH in our Galaxy manifests itself.

All these methods are indirect. They indicate only that in a small volume a large mass is concentrated. Based on the theory, General Relativity (GR), one may conclude that there is a BH inside this volume.

BHs may also be spotted by the gravitational lensing of some invisible objects. In this way the Massive Astrophysical Compact Halo Objects (MACHOs) have been discovered. One, of course, cannot exclude that these invisible objects are e.g. some low luminosity stars.

Because of all that some skepticism concerning an existence of $\mathrm{BH}$ was not eliminated, but recently it was strongly hit by the registration of gravitational waves (LIGO and Virgo) and by the photo of a black hole shadow (see below).

The form of the signal created by gravitational waves is best fit to the hypothesis that it is emitted by a coalescing BH binary with the Schwarzschild metric of each BH. Masses of both initial $\mathrm{BH}$ and the final one are accurately determined and their spins are measured. These are the first observations proving the validity of GR for strong gravitational fields. All numerous earlier tests were performed only for very weak fields.

The Event Horizon Telescope Collaboration have made a snapshot of BH shadow [4] in full agreement with the GR predictions. The photographed $\mathrm{BH}$ is situated in giant elliptical galaxy M87, at the distance $16.4 \mathrm{Mpc}$. The measured BH mass is huge, $M=(6.5 \pm 0.7) \cdot 10^{9} M_{\odot}$, It is the most massive $\mathrm{BH}$ registered in the present day universe, at the redshift $z=0$.

\subsection{GR solutions predicting black holes}

According to General Relativity four and only four different types of BHs can exist. These solutions are characterized by three parameters, the so called hairs, which can be measured through the gravitational and electric fields by an observer outside BH. These "hairs" are the BH mass $M$, its electric charge $Q$, and the angular momentum, spin, characterized by the dimensionless parameter $a$, which is confined in the limits: $0<a<1$.

There are four types of the (analytic!) solutions of the General Relativity equations describing all four possible types of BHs:

- Schwarzschild solution (1916), the simplest BH with $Q=a=0$;

- Reissner-Nordström solution (1916,1918), with $Q \neq 0$, but $a=0$;

- Kerr solutiion (1963) with $a \neq 0$ but $Q=0$;

- Kerr-Newman solution (1965) $Q \neq 0$ and $a \neq 0$.

Note that if the photon mass is nonzero, $m_{\gamma} \neq 0$, whatever tiny, electric hairs do not exist. Coulomb field around electrically charged BH completely disappears. There is no continuous transition from $m_{\gamma} \neq 0$ to $m_{\gamma}=0$. 
The metric around the Schwarzschild black hole with mass $M$ has the form:

$$
d s^{2}=\eta d t^{2}-(1 / \eta) d r^{2}-r^{2} d \theta^{2}-r^{2} \sin ^{2} \theta d \phi^{2}
$$

where $\eta=1-r_{g} / r$, with the gravitational radius (or Schwartzschildt horizon) equal to $r_{g}=$ $2 \mathrm{M} / \mathrm{m}_{P l}^{2}$ and $m_{P l} \approx 1.2 \cdot 10^{19} \mathrm{GeV}$ is the Planck mass. In the natural system of units (see Sec. 5) $1 / m_{P l}^{2}$ is equal to the Newtonian gravitational coupling constant.

This metric has a striking property that an object falling into a BH never cross the horizon (by the clock of a distant observer). That's why Zeldovich and Novikov called black holes as frozen stars.

\subsection{Black holes by creation mechanisms}

There are three possible types of $\mathrm{BH}$ creation and accordingly the black holes have the names: astrophysical BHs, supermassive BHs in galactic centers (SMBH), and primordial black holes PBH), though such a devision is rather arbitrary.

- Astrophysical black holes, which are created by stellar collapse, when star exhausted its fuel and the internal pressure became so weak that it could not resist gravity. Masses of such BHs are expected expected to be just above the neutron star masses, $3 M_{\odot}$, and normally quite close to it. Instead the observed mass spectrum of BHs in the Galaxy has maximum at $M \approx 8 M_{\odot}$ with the width: $\sim(1-2) M_{\odot}$ (see below). There is no convincing explanation of these data.

- BH crated by accretion of matter to the regions with excessive matter density. Presumably in any large galaxy there is a supermassive $\mathrm{BH}(\mathrm{SMBH})$. The masses of these black holes are in the range from billions solar masses, $M \sim 10^{9} M_{\odot}$ in elliptic and lenticular galaxies and $M \sim\left(10^{6}-10^{7}\right) M_{\odot}$ in elliptic galaxies, like Milky Way.

However, the known mechanisms of accretion is not efficient enough to create such monsters during the available time equal to the universe age $t_{U} \approx 15 \mathrm{Gyr}$. A longer, more than by an order of magnitude, time duration is necessary. Moreover SMBH are found in very small galaxies and one SMBH lives even in almost empty space. SMBH are also discovered recently in quite young universe with the age about $0.5-1$ Gyr. Their formation is multifold less probable. Massive seed are necessary, but their origin is mysterious.

It is tempting to conclude that the SMBH are primordial and type of the galaxy is determined by the mass of $\mathrm{BH}$ and not vice versa.

- Primordial black holes $(\mathrm{PBH})$ created at pre-stellar epoch in the very early universe. The canonical picture of their creation is the following. It might happen in the early universe that the density contrast was accidentally very large, $\delta \rho / \rho \sim 1$, at the cosmological horizon scale. Normally $\delta \rho / \rho \sim 10^{-4}$, but large fluctuations at small scales are not excluded. Such a piece of volume would be inside its gravitational radius, so it decoupled from the cosmological expansion and became a black hole. Such a scenario of PBH formation has been suggested by Zeldovich and Novikov [5] and elaborated later by Carr and Hawking [6].

Usually this mechanism leads to creation of PBHs with rather low masses and with sharp almost delta-function mass spectrum. A different mechanism suggested in our paper with J. Silk [1] (see also [2]) could make PBH with masses exceeding millions solar masses and with the extended log-normal mass spectrum which became quite popular recently. 


\section{Puzzles of the present day universe}

\subsection{Supermassive black holes today}

Every large galaxy and even several smaller ones contain a central supermassive $\mathrm{BH}$ with mass larger than $10^{9} M_{\odot}$ in giant elliptical and compact lenticular galaxies and $\sim 10^{6} M_{\odot}$ in spiral galaxies like Milky Way. The most massive out of them is the recently observed BH with the mass mass $(6.5 \pm 0.7) \cdot 10^{9} M_{\odot}$, see subsection 2.1. The origin of these BHs is not understood. The accepted faith is that these BHs are created by matter accretion to a central seed. But, the usual accretion efficiency is insufficient to create them during the Universe life-time, $14.6 \mathrm{Gyr}$. According to the calculations of ref. [7] to create the supermassive black hole SgrA* with the mass $\sim 4 \times 10^{6} M_{\odot}$ at the centre of our Galaxy the mean accretion rate of $4 \times 10^{-4} M_{\odot}$ per year is required. At present, X-ray observations constrain the rate of hot gas accretion to $\dot{M} \sim 3 \times 10^{-6} M_{\odot}$ per year and polarization measurements constrain it near the event horizon to $\dot{M}_{\text {horizon }} \sim 10^{-8} M_{\odot} / \mathrm{yr}$. Thus the universe age is short by two orders of magnitude.

Even more puzzling: SMHBs are observed in small galaxies and even in almost EMPTY space, where the material to make a SMBH cannot be found.

There are a few more pieces of evidence indicating that the conventional picture of SMBH formation is not compatible with observations. The mass of $\mathrm{BH}$ is typically $0.1 \%$ of the mass of the stellar bulge of galaxy [8,9] but some galaxies may have huge BH: e.g. NGC 1277 has the central $\mathrm{BH}$ of $1.7 \times 10^{10} M_{\odot}$, or $60 \%$ of its bulge mass. [10]. This creates serious problems for the standard scenario of the formation of central supermassive BHs by accretion of matter in the central part of a galaxy.

Hence an inverted picture looks more plausible, when first a supermassive $\mathrm{BH}$ was formed and attracted matter being a seed for subsequent galaxy formation, as it is suggested in refs. [1, 2, 10].

A really striking piece of evidence in favor of creation of SMBH prior to the galaxy formation is a discovery of "A Nearly Naked Supermassive Black Hole"[11] with the host galaxy having much smaller mass than the SMBH.

There are some more systems of multiple SMBH in close vicinity, which is highly improbable in the conventional model. There are:

- four binaries of SMBH discovered during recent years [12, 13, 14, 15].

- a physical association of four quasars at $z \approx 2$ [16]. The probability of finding a quadruple quasar in such close vicinity is $\sim 10^{-7}$.

- triple quasar [17]. Such a structure can only satisfy one of the three scenarios: a triple supermassive black hole (SMBH) interacting system, a triple AGN, or a recoiling SMBH.

An orthodox point of view for the explanation of appearance of such structures is merging of two spiral galaxies creating an elliptical galaxy, leaving two or more SMBHs in the center of the merged elliptical. No other way is found in the traditional approach. However, even one SMBH is hard to create. Heretic but simpler suggestion is that primordial SMBH formed binaries in the very early universe and seeded galaxy formation

\subsection{Intermediate mass black holes}

Intermediate mass black holes (MBH) are those with the masses in the interval $M=\left(10^{3}-10^{5}\right) M_{\odot}$. Nobody expected them and now they came out as if from cornucopia (cornu copiae). According 
to our conjecture [18] the intermediate mass BHs: with $M \sim 10^{3} M_{\odot}$, seeded globular clusters and IMBH with $M \sim 10^{4}-10^{5}$ seeded dwarf galaxies. Three years ago only $10 \mathrm{IMBH}$, were known with masses. $M=3 \times 10^{4}-2 \times 10^{5} M_{\odot}$. Forty of them, but somewhat more mssive, are found recently by Chandra with $10^{7}<M<3 \cdot 10^{9}[19]$ and more and more are appearing in different mass intervals with an impressive rate. In ref. [20] the discovery of 204 black holes with masses in the range $(1-20) \times 10^{5} M_{\odot} . "$ is reported. The group [21] identified a sample of $305 \mathrm{IMBH}$ candidates with $3 \times 10^{4}<M_{\mathrm{BH}}<2 \times 10^{5} M_{\odot}$, residing in galaxy centers and are accreting gas that creates characteristic signatures of a type-I active galactic nucleus.

A very interesting example of IMBH is reported in ref. [22]. According to the authors statement an invisible massive object in the central region of our Galaxy, based on the high-resolution molecular line observations with the Atacama Large Millimeter/submillimeter Array (ALMA). The morphology and kinematics of these streams can be reproduced well through two Keplerian orbits around a single point mass of $(3.2 \pm 0.6) \times 10^{4} \mathrm{M}_{\odot}$. The results provide new circumstantial evidences for a wandering intermediate-mass black hole in the Galactic center (tramp in the galaxy), suggesting also that high-velocity compact clouds can be probes of quiescent black holes abound in our Galaxy.

It is tempting to conclude that such particular object is a primordial black hole, as well a all other IMBH, mentioned above, whose abundance is difficult to explain other way.

Only one or two massive BH are observed in Globular clusters [23, 24]. Definite evidence is presented for BH with $M \approx 2000 M_{\odot}$ found in the core of the globular cluster 47 Tucanae [23]. The standard model does not have an explanation for the origin of such relatively light IMBHs.

We assumed [18] that such IMBHs are primordial. If the parameters of the log-normal mass distribution of PBHs are chosen to fit the LIGO data and the density of SMBH, then the number of PBH with masses $(2-3) \times 10^{3} M_{\odot}$ is about $10^{4}-10^{5}$ per one SMPBH with mass $>10^{4} M_{\odot}$. This predicted density of IMBHs is sufficient to seed the formation of all globular clusters in galaxies. according to the published estimates. This density of IMBHs is sufficient to seed the formation of globular clusters in galaxies. Our prediction is that IMBHs exist in all globular clusters but it is difficult to observe them.

It is assumed that all PBH with $M>10^{4} M_{\odot}$ strongly accreted matter and grew up to billion solar masses.

\subsection{A strange galaxy}

Very recent a surprising discovery of "A young galaxy cluster in the old Universe" [25] was announced. There was discovered a blue cluster, that is a local galaxy cluster with an unprecedentedly high fraction of blue star-forming galaxies yet hosted by a massive dark matter halo. The blue fraction is 0.57 , which is $4.0 \sigma$ higher than those of the other comparison clusters under the same selection and identification criteria. The probability to find such a high blue fraction in an individual cluster is only $0.003 \%$, which challenges the current standard frameworks of the galaxy formation and evolution in the $\Lambda$ CDM Universe.

Such a blue cluster seems to be in accord with our prediction of predominantly helium rich "bubbles" in the universe. Indeed in the bubbles with high baryon-to-photon ratio the big bang nucleosynthesis could produce considerably higher abundance of the primordial helium- 4 , than the usual $25 \%$. 


\subsection{Peculiar stars in the Milky Way}

- Too old stars in the Milky Way:

During the last several years the precision in determination of the stellar age has been drastically improved since not only uranium and thorium abundances but several other elements were used as nuclear chronometers.

Employing thorium and uranium abundances in comparison with each other and with several stable elements the age of metal-poor, halo star BD $+17^{\circ} 3248$ was estimated as $13.8 \pm 4 \mathrm{Gyr}$ [26], which noticeably exceeds the age of inner halo of the Galaxy $11.4 \pm 0.7 \mathrm{Gyr}$, according to ref. [27]

The age of a star in the galactic halo, HE 1523-0901, was estimated to be about 13.2 Gyr [28] In this work first time many different chronometers, such as the U/Th, U/Ir, Th/Eu and Th/Os ratios to measure the star age have been employed.

The most striking example of stellar Methuselah is the metal deficient high velocity subgiant in the solar neighborhood HD 140283 has the age $14.46 \pm 0.31 \mathrm{Gyr}$ [29]. The central value of the age of this star exceeds the universe age by two standard deviations, if, $H=67.3$, as determined from the angular fluctuations of $\mathrm{CMB}$ and correspondingly $t_{U}=13.8$. On the other hand, according to the recent determination of $H$ by the traditional astronomical methods, $H=74$, and $t_{U}=12.5$. So the star age exceed the universe age by more than $10 \sigma$.

This cannot be so, of course, in the conventional cosmology and astrophysics but bur model [1, 2] predicts unusual initial chemical content for a small number of stars, so they may look older than they are. "Our'" stars are primordial and they may have ubusually high velocity in the Galaxy as is hinted by HD 140283. Moreover, there are more very fast stars in the Galaxy, as we see in the following subsection.

Let us mention in conclusion a very old planet in the Kepler-10 Planetary System with the age $10.6_{-1.3}^{+1.5}$ Gyr [30]. For comparison the age of the Earth is $4.54 \mathrm{Gyr}$. The planet formation is a slow process, it should be preceded by a supernova explosion, formation of molecules, and dust. However, as we see in what follows, the early universe is unexpectedly dusty.

- High velocity and unusual chemical content stars in the Galaxy

The Galaxy, as discovered recently, has quite significant number of very fast stats, with velocities higher than the escape velocity and of stars with rather surprising chemical content. The normal stellar velocity in the galaxy is in the range $100-200 \mathrm{~km} / \mathrm{sec}$. Some pulsars are observed with the velocities about $1000 \mathrm{~km} / \mathrm{sec}$. The origin of the letter is evident. Pulsars are created through the stellar collapse and even a tiny non-sphericity of the emitted neutrino flux would create huge recoil momentum, sufficient to accelerate the up to $1000 \mathrm{~km} / \mathrm{sec}$. However, the origin of the stars moving faster than galactic escape velocity is puzzling.

A discovery of high speed low-mass white dwarf (LP 40-365) that "travels at a velocity greater than the Galactic escape velocity and whose peculiar atmosphere is dominated by intermediatemass elements", is reported in ref. [31] The origin of this star is mysterious. It may be a compact primordial star, predicted by the model of refs. [1,2]

Some more references to recently discovered high velocity stars in the galaxy includes the "Old, Metal-Poor Extreme Velocity Stars in the Solar Neighborhood" [32] and a special search for very such quickly moving stars: "Gaia DR2 in 6D: Searching for the fastest stars in the Galaxy" [33]. The authors conclude that the origin of the discovered stars is unclear. They might 
be accelerated to high velocity by a population of IMBH in Globular clusters, if there is sufficient number of IMBHs.

A discovery of a very unusual star is reported in ref. [34]. The authors found red host star and planet masses of $M_{\text {host }}=0.15_{-0.10}^{+0.27} M_{\odot}$ and $m_{p}=18_{-12}^{+34} M_{\oplus}$. The life-time of main sequence star with the solar chemical content is larger than $t_{U}$ already for $M<0.8 M_{\odot}$. The origin is unknown. It may be a primordial helium star?

Another puzzling discovery of "A class of partly burnt runaway stellar remnants from peculiar thermonuclear supernovae" is announced in [35]. Discovery of three chemically peculiar runaway stars, survivors of thermonuclear explosions - according to the authors. "With masses and radii ranging between $0.20-0.28 M_{\odot}$ and $0.16-0.60 R_{\odot}$, respectively, as is stated in the paper, these inflated white dwarfs are the partly burnt remnants of either peculiar Type SNIa or electron-capture supernovae".

And at last, the discovery of this year [35]: "A hyper-runaway white dwarf in Gaia DR2 as a Type Iax supernova primary remnant candidate" Quoting the authors: "We report the likely first known example of an unbound white dwarf that is consistent with being the fully-cooled primary remnant to a Type Iax supernova. The candidate, LP 93-21, is travelling with a galactocentric velocity of $\mathrm{v}_{\text {gal }} \simeq 605 \mathrm{~km} \mathrm{~s}^{-1}$, and is gravitationally unbound to the Milky Way, We rule out an extragalactic origin. The Type Iax supernova ejection scenario is consistent with its peculiar unbound trajectory, given anomalous elemental abundances are detected in its photosphere via spectroscopic follow-up. This discovery reflects recent models that suggest stellar ejections likely occur often." This could be a peculiar WD or a remnant of a primordial star.

\subsection{MACHOs}

MACHOs is the abbreviation for Massive Astrophysical Compact Halo Objects discovered through gravitational microlensing by Macho and Eros groups. They are invisible (very weakly luminous or even non-luminous) objects masses about a half of the solar mass in the Galactic halo, in the center of the Galaxy, and recently in the Andromeda (M31) galaxy. Their density is significantly greater than the density expected from the known low luminosity stars and the BH of similar mass.

The data on the MACHO abundance presented by different groups are rather controversial. The present date situation is reviewed and summarized in refs. [36, 37, 38, 39, 40]. Briefly, the situation is the following.

MACHO group [41] reported registration of 13 - 17 microlensing events towards the Large Magellanic Cloud (LMC), which is significantly higher than the number which could originate from the known low luminosity stars. On the other hand this amount is not sufficient to explain all dark matter in the halo. The fraction of the mass density of the observed objects, which created the microlensing effects, with respect to the energy density of the dark matter in the galactic halo, $f$, according to the observations [41] is in the interval:

$$
0.08<f<0.50
$$

at $95 \% \mathrm{CL}$ for the mass range $0.15 M_{\odot}<M<0.9 M_{\odot}$.

EROS collaboration [42] has placed the upper limit on the halo fraction, $f<0.2$ (95\% CL) for the objects in the specified above MACHO mass range, while EROS-2 [43] gives $f<0.1$ for 
$0.6 \times 10^{-7} M_{\odot}<M<15 M_{\odot}$ for the survey of Large Magellanic Clouds. It is considerably less than that measured by the MACHO collaboration in the central region of the LMC.

The new analysis of 2013 by EROS-2, OGLE-II, and OGLE-III collaborations [44] towards the Small Magellanic Cloud (SMC). revealed five microlensing events towards the SMC (one by EROS and four by OGLE), which lead to the upper limits $f<0.1$ obtained at $95 \%$ confidence level for MACHO's with the mass $10^{-2} M_{\odot}$ and $f<0.2$ for MACHOs with the mass $0.5 M_{\odot}$.

Search for microlensing in the direction of Andromeda galaxy (M31) demonstrated some contradicting results [36, 37] with an uncertain conclusion. E.g. AGAPE collaboration [45], finds the halo MACHO fraction in the range $0.2<f<0.9$. while MEGA group presented the upper limit $f<0.3$ [46]. On the other hand, the recent discovery of 10 new microlensing events [47] is very much in favor of MACHO existence. The authors conclude: "statistical studies and individual microlensing events point to a non-negligible MACHO population, though the fraction in the halo mass remains uncertain".

Some more recent observational data and the other aspects of the microlensing are discussed in ref. [48].

It would be exciting if all DM were constituted by old stars and black holes made from the high density baryon bubbles as suggested in refs. $[1,2]$ with masses in still allowed intervals, but more detailed analysis of this possibility has to be done.

There is a series of papers claiming the end of MACHO era. For example in ref. [49] the authors stated "we exclude MACHOs with masses $M>43 M_{\odot}$ at the standard local halo density. This removes the last permitted window for a full MACHO halo for masses $M>10^{-7.5} M_{\odot}$.

In addition to the criticism raised in the paper [49], some more arguments against abundant galactic population of MACHOs are also presented in ref. [50, 51]. However, according to the paper [52], the approach of the mentioned works have serious flaws and so their results are questionable. A reply to this criticism is presented in the subsequent paper [53].

The data in support of smaller density of MACHOs in the direction to SMC is presented in ref. [43]

Later, however, another paper of the Cambridge group [54] was published where, on the basis of studies of binary stars, arguments in favor of real existence of MACHOs and against the pessimistic conclusions of ref. [49] were presented.

The latest investigation on the "end of MACHO era" was presented in ref. [55], where it is concluded that "the upper bound of the MACHO mass tends to less than $5 M_{\odot}$ does not differ much from the previous one. Together with microlensing studies that provide lower limits on the MACHO mass, our results essentially exclude the existence of such objects in the galactic halo".

A nice review of the state of the art and some new data is presented in ref. [47] with the conclusion that some statistical studies and individual microlensing events point to a non-negligible MACHO population, though the fraction in the halo mass remains uncertain.

According to the results of different groups the fraction of MACHO mass density with respect to the total mass density of dark matter varies in rather wide range:

$$
f_{M A C H O}=\frac{\rho_{M A C H O}}{\rho_{D M}} \sim(0.01-0.1)
$$

Notice a large variance of the results by different groups. 
An interesting option is that the spatial distribution of MACHOs may be very inhomogeneous and non-isotropic. Due to selection effect, MACHOs are observed only in over-dense clumps where their density is much higher than the average one. For a review and the list of references on dark matter clumping see e.g. [56]. Clumping of primordial back holes, due to dynamical friction, may be much stronger than the clumping of dark matter consisting of elementary particles. This hypothesis would allow to avoid contradiction between the observed high density of MACHOs and the predicted much smaller density of them based on the log-normal mass spectrum with $M_{m}=$ $(7-9) M_{\odot}$.

\subsection{Mass spectrum of astrophysical BH in the Galaxy}

It was discovered during the last decade that the $\mathrm{BH}$ masses in the Galaxy are concentrated in the narrow range $(7.8 \pm 1.2) M_{\odot}$ [57]. This result agrees with another paper where a peak around $8 M_{\odot}$, a paucity of sources with masses below $5 M_{\odot}$, and a sharp drop-off above $10 M_{\odot}$ are observed [58], These features are not easily explained in the standard model of $\mathrm{BH}$ formation by stellar collapse.

\subsection{Gravitational waves from $\mathrm{BH}$ binaries}

Registration of gravitational waves (GW) from coalescing BH binaries solved long existed problems of test of GR for strong field and presented a first direct proof of BH existence. However, it opens immediately several more problems. "In much wisdom is much grief". In short there are three very interesting problems addressed in our paper [39]:

- 1. Origin of heavy BHs with the masses $\sim 30 M_{\odot}$.

- 2. Formation of BH binaries from the original stellar binaries.

- 3. Low spins of the coalescing BHs

1. Massive BH origin. Such BHs are believed to be created by massive star collapse, though a convincing theory is still lacking. To form so heavy BHs, the progenitors should have $M>100 M_{\odot}$ i.e. the masses two orders of magnitude higher than the solar mass and a low metal abundance to avoid too much mass loss during the evolution. Such heavy stars might be present in young star-forming galaxies but they are not observed in the necessary amount.

Mass of BH in LIGO binaries is up to $50 M_{\odot}$ (surprise, but can be reconciled with stellar evolution), rumors that a $100 M_{\odot}$ was found - a puzzle for stellar evolution. (borrowed from K.Postnov talk at Isaak Markovich Khalatnikov Centennial Conference).

On the other hand, primordial BH with the observed by LIGO masses may be created with sufficiently high density.

2. Formation of $B H$ binaries. Stellar binaries were formed from common interstellar gas clouds and are quite frequent in galaxies. If $\mathrm{BH}$ is created through stellar collapse, a small nonsphericity leads to a very large recoil velocity of the $\mathrm{BH}$ and the binary is destroyed. An example of such effect is demonstrated by huge velocities of pulsars in the Galaxy.) BH formation from PopIII stars and subsequent formation of BH binaries with $(36+29) M_{\odot}$ is analyzed and found to be negligible.

The problem of the binary formation is simply solved if the observed sources of GWs are the binaries of primordial black holes ( $\mathrm{PBH})$. They were at rest in the comoving volume and may have non-negligible probability to become gravitationally bound. 
3. The low value of the BH spins in GW150914 and in almost all (except for three) other events. It strongly constrains astrophysical $\mathrm{BH}$ formation from close binary systems. Astrophysical BH are expected to have considerable angular momentum but still the dynamical formation of double massive low-spin BHs in dense stellar clusters is not excluded, though difficult. On the other hand, PBH practically do not rotate because vorticity perturbations in the early universe are known to be vanishingly small.

However, individual PBH forming a binary initially rotating on elliptic orbit could gain collinear spins about $0.1-0.3$, rising with the PBH masses and eccentricity $[59,60]$ This result is in agreement with the GW170729 LIGO event produced by the binary with masses $50 M_{\odot}$ and $30 M_{\odot}$ and probably with GW151216. Earlier in the works [61, 62] much weaker gain of the angular momentum gain was obtained.

\section{Surprises of the Early Universe}

The data collected during last several years indicate that the young universe at $z \sim 10$ is grossly overpopulated with unexpectedly high amount of:

\subsection{Superluminous young galaxies}

Several galaxies have been observed at high redshifts, with natural gravitational lens "telescopes, e.g. a galaxy at $z \approx 9.6$ which was created when the universe was about 0.5 Gyr old [63] Moreover, galaxy at $z \approx 11$ has been detected which was formed earlier than the universe age was $0.41 \mathrm{Gyr}$ (or even shorter with larger $\mathrm{H}$ ). This galaxy is three times more luminous in UV than other galaxies at $z=6-8$. The discovery of another unexpectedly early created galaxy at red-shift $z=11$ is reported in ref. [66].

The discovery of not so young but extremely luminous galaxy with the luminosity $L=3 \cdot 10^{14} L_{\odot}$ was announced in ref. [65].This galaxy already existed, when the universe age was $t_{U} \sim 1.3 \mathrm{Gyr}$. The galactic seeds, or embryonic black holes, necessary for the creatkon of such a huge galaxy might be bigger than thought possible. Ona of the authors of the work P. Eisenhardt: said "How do you get an elephant? One way is start with a baby elephant." The BH was already billions of $M_{\odot}$, when our universe was only a tenth of its present age of 13.8 billion years. "Another way to grow this big is to have gone on a sustained binge, consuming food faster than typically thought possible." A low spin of the seed is necessary.

To finish with the list of these surprises let us mention almost yesterday discovery, reported in ref. [67], of the submillimeter (wavelength 870um) detections of 39 massive star-forming galaxies at $z>3$, which are unseen in the spectral region from the deepest ultraviolet to the near-infrared. They contribute a tota star-formation-rate density ten times larger than that of equivalently massive ultraviolet-bright galaxies at $z>3$. Residing in the most massive dark matter halos at their redshifts, they are probably the progenitors of the largest present-day galaxies in massive groups and clusters. Such a high abundance of massive and dusty galaxies in the early universe challenges our understanding of massive-galaxy formation.

An onset of the star formation 250 million years after the Big Bang is claimed in ref. [68]. The authors observed the oxygen line at $z=9.1096 \pm 0.0006$. According to their conclusion "This precisely determined redshift indicates that the red rest-frame optical colour arises from a dominant 
stellar component that formed about 200 million years after the Big Bang, corresponding to a redshift of about 15. Although we are observing a secondary episode of star formation at $z=9.1$, the galaxy formed the bulk of its stars at a much earlier epoch."

As is stated in the paper "Monsters in the Dark" [69], the density of galaxies at $z \approx 11$ is $10^{-6}$ $\mathrm{Mpc}^{-3}$, an order of magnitude higher than estimated from the data at lower $\mathrm{z}$. The origin of these galaxies is unclear.

According to ref. [70] "Rapid emergence of high-z galaxies so soon after big bang may actually be in conflict with current understanding of how they came to be. This problem is very reminiscent of the better known (and probably related) premature appearance of supermassive black holes at $z \sim 6$. It is difficult to understand how $10^{9} M_{\odot}$ black holes appeared so quickly after the big bang without invoking non-standard accretion physics and the formation of massive seeds, both of which are not seen in the local Universe."

\subsection{Supermassive BH and/or QSO}

Another and even more striking example of early formed objects are high z quasars. About 40 quasars with $z>6$ were known three years ago, each quasar containing BH with $M \sim 10^{9} M_{\odot}$.

The maximum redshift QSO is discovered in ref. [71] at the redshift $z=7.085$, luminocity $L \approx 6 \cdot 10^{13} L_{\odot}$, and the mass $M=2 \cdot 10^{9} M_{\odot}$, The quasar was formed before the universe reached $0.75 \mathrm{Gyr}$.

In addition to this forty quasars, one more monster was discovered [72] with the outrageously huge mass $1.2 \cdot 10^{10} M_{\odot}$. There is already a serious problem with formation of lighter and less luminous quasars which is multifold deepened with this new "creature". The new one with more than 10 billion solas masses is absolutely forbidden in the standard approach.

Recent observations by SUBARU practically doubled the number of discovered high z quasars [74] In particular this group reported the first low luminosity QSO at $z>7$.

The accretion rate, as estimated in ref. [73] is about $2200 M_{\odot}$ during $320 \mathrm{Myr}$ in a halo with a mass of $3 \times 10^{10} M_{\odot}$ at $z=7.5$. It is by far less than is necessary.

The observation of a 800 million solar mass black hole in a significantly neutral universe at redshift 7.5 [75] indicated that accretion in this particular case is absent, otherwise the plasma should be ionized.

It is difficult to understand how $10^{9} M_{\odot}$ black holes (to say nothing about $10^{10} M_{\odot}$ ) appeared so quickly after the big bang without invoking non-standard accretion physics and the formation of massive seeds, both of which are not seen in the local Universe.

To conclude on QSO/SMBH:

The quasars are supposed to be supermassive black holes and their formation in such short time by conventional mechanisms looks problematic, to say the least.

Such black holes, formed when the Universe was less than one billion years old, present substantial challenges to theories of the formation and growth of black holes and the coevolution of black holes and galaxies.

Even the origin of SMBH in contemporary universe during $14 \mathrm{Gyr}$ is difficult to explain. Nonstandard accretion physics and the formation of massive seeds seem to be necessary. Neither of them is observed in the present day universe. 


\subsection{Evolved chemistry, dust, supernovae, and gamma-bursters}

The medium around the observed early quasars contains considerable amount of "metals" (elements heavier than $\mathrm{He}$ ). According to the standard picture, only elements up to ${ }^{4} \mathrm{He}$ and traces of $\mathrm{Li}, \mathrm{Be}, \mathrm{B}$ were formed by big bang hucleosynthesis $(\mathrm{BBN})$, which took place when the universe was about a 100 second old. The heavier elements, according to the conventional cosmology, were created much later by stellar nucleosynthesis and dispersed in the interstellar space by supernova explosions. Hence, an evident but not necessarily true conclusion was that prior to or simultaneously with the QSO formation a rapid star formation should take place. These stars should evolve to a large number of supernovae enriching interstellar space by metals through their explosions.

Another possibility is a non-standard BBN in bubbles with very high baryonic density, which allows for formation of heavy elements beyond lithium in the very early universe, as suggested in refs $[1,2]$.

The universe at $z>6$ is not only enriched by metals but also is quite dusty, as observed in the papers [76, 77]. Abundant dust is observed in several early galaxies, e.g. in HFLS3 at $z=6.34$ and in A1689-zD1 at $z=7.55$ [78]. Copious Amounts of Dust and Gas is seen in a $z=7.5$ Quasar Host Galaxy [79]. Dusty galaxies show up at redshifts corresponding to a Universe which is only about 500 Myr old. Very high past star formation is needed to explain the presence of $\sim 10^{8} M_{\odot}$ of dust implied by the observations [80]. .

Later made catalogue of the observed dusty sources [81] confirms earlier observations and indicates that the number of dusty sources is an order of magnitude larger than predicted by the canonical theory.

An analysis of the observations and a review of possible scenarios of dust dust production in galaxies at $z \sim 6-8.3$ is presented in [82]. The authors conclude that the mechanism of dust formation in galaxies at high redshift is still unknown. Asymptotic giant branch (AGB) stars and explosions of supernovae ( $\mathrm{SNe}$ ) are possible dust producers, and non-stellar processes may substantially contribute to dust production. However, AGB stars are not efficient enough to produce the amounts of dust observed in the galaxies.

In order to explain these dust masses, SNe would have to have maximum efficiency and not destroy the dust which they formed. Therefore, the observed amounts of dust in the galaxies in the early universe were formed either by efficient supernovae or by a non-stellar mechanism, for instance the grain growth in the interstellar medium.

Or non-standard big bang nucleosynthesis with large baryon-to- $\gamma$ ratio leading to abundant formation of heavy elements, see below.

To make dust a long succession of processes is necessary: first, supernovae explode to deliver heavy elements into space (metals), then metals cool and form molecules, and lastly molecules make dust which could form macroscopic pieces of matter, turning subsequently into early rocky planets. Note in brackets that we all are dust from SN explosions, at much later time.

Existence of heavy elements, molecules, and dust in the early universe indicates but there already could be life. Several hundred million years may be enough for birth of living creatures.

Observations of high redshift gamma ray bursters (GBR) also indicate a high abundance of supernova at large redshifts. The highest redshift of the observed GBR is 9.4 and there are a few more GBRs with smaller but still high redshifts. 
Dust production scenarios in galaxies at high $z=6-8.3$ was reviewed in ref. [82]. The authors concluded that the mechanism of dust formation in galaxies at high redshift is still unknown. Asymptotic giant branch (AGB) stars and explosions of supernovae ( $\mathrm{SNe}$ ) are possible dust producers, and non-stellar processes may substantially contribute to dust production. However, AGS are not efficient enough to produce the amounts of dust observed in the galaxies.

In order to explain these dust masses, $\mathrm{SNe}$ would have to have maximum efficiency and not destroy the dust which they formed. Therefore, the observed amounts of dust in the galaxies in the early universe were formed either by efficient supernovae or by a non-stellar mechanism, for instance the grain growth in the interstellar medium.

Observations of high redshift gamma ray bursters (GBR) also indicate a high abundance of supernova at large redshifts [83]. The highest redshift of the observed GBR is 9.4 and there are a few more GBRs with smaller but still high redshifts.

The necessary star formation rate for explanation of these early GBRs is at odds with the canonical star formation theory. Non-standard big bang nucleosynthesis with large baryon-tophoton ratio leading to formation of heavy elements in the very early universe may be needed.

These facts are in good agreement with the our 1993 model [1] but in tension with the Standard Cosmological Model.

\section{Possible mechanism explaining the surprises in the data}

In 1993 we suggested a model AD-JS of PBH creation which allowed an abundant formation of very massive black holes with the log-normal mass spectrum:

$$
\frac{d N}{d M}=\mu^{2} \exp \left[-\gamma \ln ^{2}\left(M / M_{0}\right)\right]
$$

depending only on three constant parameters: $\mu, \gamma, M_{0}$. It can be easily generalized to a superposition of several log-normal spectra with several separated maxima.

Here we use the natural system of units where the speed of light, the reduced Planck constant, and the Boltzmann constant are all equal to unity: $c=h /(2 \pi)=k=1$. In this system the Newtonian gravitational constant is $G_{N}=1 / m_{P l}^{2}$, where th Planck mass is $m_{P l}=1.22 \cdot 10^{19} \mathrm{GeV}$.

Such a simple form pf the PBH mass spectrum is a result result of quantum diffusion of baryonic scalar field during inflation. Probably such spectrum is a general consequence of diffusion.

Log-normal mass spectrum of PBHs was rediscovered later in ref. [84]. Now in many works such spectrum is postulated without any justification. In our model the PBH creation was based on the supersymmetry (SUSY) motivated baryogenesis, proposed by Affleck and Dine (AD) [85]. SUSY predicts existence of scalars with non-zero baryon number, $B \neq 0$. Such scalar bosons may condense along flat directions of the quartic potential:

$$
U_{\lambda}(\chi)=\lambda|\chi|^{4}(1-\cos 4 \theta)
$$

and/or of the mass term, $m^{2} \chi^{2}+m^{* 2} \chi^{* 2}$ :

$$
U_{m}(\chi)=m^{2}|\chi|^{2}[1-\cos (2 \theta+2 \alpha)]
$$


where $\chi=|\chi| \exp (i \theta)$ and $m=|m| e^{\alpha}$. If $\alpha \neq 0$, charge symmetry (between particles and antiparticles) $\mathrm{C}$ and $\mathrm{CP}$ are broken.

In Grand Unified version of SUSY baryonic number is naturally non-conserved, which is reflected in non-invariance of $U(\chi)$ w.r.t. phase rotation.

Initially (after inflation) $\chi$ is normally away from the origin and, when inflation is over, starts to evolve down to the equilibrium point, $\chi=0$, Its evolution is described by the equation similar to that governing the motin of a point-like particle in Newtonian mechanics:

$$
\ddot{\chi}+3 H \dot{\chi}+U^{\prime}(\chi)=0 .
$$

Baryonic charge of $\chi$ is equal to :

$$
B_{\chi}=\dot{\theta}|\chi|^{2} .
$$

Due to instability of effectively massless field in De Sitter like stage $\chi$ can acquire a large baryonic namber. It is analogous to mechanical angular momentum in two dimensional complex $\chi$-plane. The $\chi$ decays transferred the accumulated baryonic charge to that of quarks in B-conserving process. AD baryogenesis could lead to baryon asymmetry of order of unity, much larger than the observed $10^{-9}$.

If $m \neq 0$, the angular momentum, B, can generated by a different direction of the quartic and quadratic valleys at low $\chi$. If CP-odd phase $\alpha$ is small but non-vanishing, both baryonic and antibaryonic domains might be formed with possible dominance of one of them.

Matter and antimatter domains may exist but globally $B \neq 0$.

We slightly generalized the original Affleck-Dine model introducing a general renormalizable coupling of $\chi$ to the inlfaton field $\Phi$ (the first term in the equation below):

$$
U=g|\chi|^{2}\left(\Phi-\Phi_{1}\right)^{2}+\lambda|\chi|^{4} \ln \left(\frac{|\chi|^{2}}{\sigma^{2}}\right)+\lambda_{1}\left(\chi^{4}+\text { h.c. }\right)+\left(m^{2} \chi^{2}+\text { h.c. }\right) .
$$

The other terms in this potential presents the usual Affleck-Dine potential with one loop radiation corrections. We assume that the inflaton $\Phi$ passes through the value $\Phi_{1}$ still during inflation but not too close to the end of it.

If $\Phi$ is close to $\Phi_{1}$ the window in the potential to the flat directions is open and $\chi$ can quantumfluctuate to acquire rather high amplitude. It evolves according to quantum diffusion equation derived by Starobinsky, generalized to a complex field $\chi$.

If the window to flat direction, when $\Phi \approx \Phi_{1}$ is open only during a short period, cosmologically small but possibly astronomically large bubbles with high $\beta$ could be created, occupying a small fraction of the universe, while the rest of the universe has normal $\beta \approx 6 \cdot 10^{-10}$, created by small $\chi$.

Initially the bubble with large $\beta$ would have have the almost the same density as the average cosmological density because before the QCD phase transition quarks are essentially massless and the initial perturbations are the isocurvature ones, though they might have quite large amplitude. Only after the QCD phase transition when massless quarks turn into heavy nucleons the original isocurvature perturbations transformed into density perturbations. The bubbles with high baryonic number can either form PBH or compact stellar like objects, depending on their magnitude and size. 
Such mechanism of massive PBH formation is very much different from other ones studied in the literature. The fundament of PBH creation is build at inflation by making large isocurvature fluctuations at relatively small scales, with practically vanishing density perturbations. Initial isocurvature perturbations are in chemical content of massless quarks. Density perturbations are generated rather late after the QCD phase transition.

The emerging universe looks like a piece of Swiss cheese, where holes are high baryonic density objects occupying a minor fraction of the universe volume.

The outcome, depending on $\beta=n_{B} / n_{\gamma}$, could be:

- PBHs with log-normal mass spectrum.

- Compact stellar-like objects, as e.g. cores of red giants.

-Disperse hydrogen and helium clouds with (much) higher than average $n_{B}$ density.

- $\beta$ may be negative leading to compact antistars which could survive annihilation with the homogeneous baryonic background.

A modification of inflaton interaction with scalar baryons as e.g.

$$
U \sim|\chi|^{2}\left(\Phi-\Phi_{1}\right)^{2}\left(\left(\Phi-\Phi_{2}\right)^{2}\right.
$$

gives rise to a superposition of two log-normal spectra or multi-log.

\section{Conclusion}

- 1. Natural baryogenesis model leads to abundant formation of PBHs and compact stellar-like objects in the early universe after QCD phase transition, $t \gtrsim 10^{-5} \mathrm{sec}$.

- 2. These objects had originally log-normal mass spectrum, though it may be subsequently distorted due to matter accretion, especially for very heavy ones.

- 3. PBHs formed at this scenario can explain the peculiar features of the sources of GWs observed by LIGO.

- 4. The considered mechanism solves the numerous mysteries of $z \sim 10$ universe: abundant population of supermassive black holes, early created gamma-bursters and supernovae, early bright galaxies, and evolved chemistry including dust.

- 5. There is persuasive data in favor of the inverted picture of galaxy formation, when first a supermassive BH seeds are formed and later they accrete matter forming galaxies.

- 6. Inverted picture of galaxy formation is advocated.

- 7. An existence of supermassive black holes observed in all large and some small galaxies and even in almost empty environment is naturally explained.

- 8. "Older than $t_{U}$ " stars may exist; the older age is mimicked by the unusual initial chemistry.

- 9. Existence of high density invisible "stars" (machos) is understood.

- 10. Explanation of origin of BHs with $2000 M_{\odot}$ in the core of globular cluster and the observed density of GCs is presented.

- 11. A large number of the recently observed IMBH was predicted.

-12. A large fraction of dark matter or $100 \%$ can be made of PBHs.

13. Clouds of matter with high baryon-to-photon ratio may exist.

- 14. A possible by-product: plenty of (compact) anti-stars, even in the Galaxy, not yet excluded by observations. 
Extreme claims:

- Black holes in the universe are mostly primordial.

- Primordial BHs make all or dominant part of dark matter.

- All QSO were created in the very early universe.

- Metals and dust are made much earlier than at $z=10$.

- Inverted picture of galaxy formation: seeding of galaxies by SMPBH or IMPBH;

- Seeding of globular clusters by $10^{3}-10^{4} \mathrm{BHs}$, dwarfs by $10^{4}-10^{5} \mathrm{BH}$.

\section{References}

[1] A. Dolgov, J. Silk, Baryon isocurvature fluctuations at small scales and baryonic dark matter, Phys.Rev. D47 (1993) 4244.

[2] A.D. Dolgov, M. Kawasaki, N. Kevlishvili, Inhomogeneous baryogenesis, cosmic antimatter, and dark matter, Nucl.Phys. B807 (2009) 229 [hep-ph/0806.2986].

[3] A.D. Dolgov, Massive and supermassive black holes in the contemporary and early Universe and problems in cosmology and astrophysics, Usp.Fiz.Nauk, 188 (2018) 121; Phys.Usp. 61 (2018) 115 [astro-ph/1705.06859].

[4] Event Horizon Telescope Collaboration (K. Akiyama et al) First M87 Event Horizon Telescope Results. IV. Imaging the Central Supermassive Black Hole, Astrophys.J. 875 (2019) L4 [astro-ph.GA/1906.11240].

[5] Ya,B. Zeldovich, I.D. Novikov, The Hypothesis of Cores Retarded during Expansion and the Hot Cosmological Model, Sov. Astron., 10 (1967), 602.

[6] B.J. Carr, S.W. Hawking, Black holes in the early Universe, MNRAS, 168 (1974), 399.

[7] E.M. Murchikova, E.S. Phinney, A. Pancoast, R.D. Blandford, A Cool Accretion Disk around the Galactic Centre Black Hole, Nature, 570 (2019) 83.

[8] Häring N., Rix H.-W., On the Black Hole Mass - Bulge Mass Relation, Astrophys. J. Lett. 604 (2004), L89 [astro-ph/0402376]

[9] E. Sani, et al, The Spitzer/IRAC view of black hole - bulge scaling relations, Mon. Not. R. Astron. Soc., 413 (2011) 1479. [asro-ph/1012.3073].

[10] R.C.E, van den Bosch, et al., Nature, 491 (2012) 729 [asro-ph/1211.6429].

[11] J.J. Condon, et al A Nearly Naked Supermassive Black Hole. The Astrophysical Journal, 834 (2017) 184 [astro-ph/1606.04067].

[12] P. Kharb, et al A candidate sub-parsec binary black hole in the Seyfert galaxy NGC 7674, published in Nature Astronomy, [asrto-ph/1709.06258].

[13] C. Rodriguez et al. A compact supermassive binary black hole system. Ap. J. 646 (2006) 49 [astro-ph/0604042].

[14] M.J. Valtonen, New orbit solutions for the precessing binary black hole model of OJ 287, Ap.J. 659 (2007) 1074

[15] M.J. Graham et al. A possible close supermassive black-hole binary in a quasar with optical periodicity, Nature, 518 (2015) 74 [astro-ph/1501.01375 ]. 
[16] J.F. Hennawi J.F., et al, Quasar quartet embedded in giant nebula reveals rare massive structure in distant universe, Science, 348 (2015), 779.

[17] E. Kalfountzou, M.S. Lleo, M. Trichas, SDSS J1056+5516: A Triple AGN or an SMBH Recoil Candidate?" [astro-ph/1712.03909].

[18] A. Dolgov, K. Postnov, Globular Cluster Seeding by Primordial Black Hole Population, JCAP 1704 (2017) 036, [astro-ph/1702.07621].

[19] M. Mezcua, et al Intermediate-mass black holes in dwarf galaxies out to redshift ÂćÂL̊ ŠS 2.4 in the Chandra COSMOS Legacy Survey, [astro-ph/1802.01567].

[20] He-Yang Liu, et al, it A Uniformly Selected Sample of Low-Mass Black Holes in Seyfert 1 Galaxies [astro-ph/1803.04330].

[21] I.V. Chilingarian, et al. A Population of Bona Fide Intermediate Mass Black Holes Identified as Low Luminosity Active Galactic Nuclei [astro-ph/1805.01467].

[22] S. Takekawa, et al., Indication of Another Intermediate-mass Black Hole in the Galactic Center, [astro-ph/1812.10733].

[23] B. Kiziltan, H. Baumgardt, A. Loeb, An intermediate-mass black hole in the centre of the globular cluster 47 Tucanae, Nature, 542 (2017) 203 [[astro-ph/1702.02149].

[24] Perera B.B.P., et al., Evidence for an intermediate-mass black hole in the globular cluster NGC 6624, MNRAS, 468 (2017) 2114 [astro-ph/1705.01612].

[25] T. Hashimoto, et al, A young galaxy cluster in the old Universe, [astro-ph/1908.01666].

[26] .J. Cowan, et al The chemical composition and age of the metal-poor halo star BD+17 3248 , Ap.J. 572 (2002) 861 [astro-ph/0202429].

[27] J. Kalirai, The Age of the Milky Way Inner Halo, Nature, 486 (2012) 90 [astro-ph/1205.6802].

[28] A. Frebe, et al Discovery of HE 1523-0901: A Strongly r-Process Enhanced Metal-Poor Star with Detected Uranium, Astrophys.J. 660 (2007) L117 [astro-ph/0703414].

[29] H. E. Bond, et al, HD 140283: A Star in the Solar Neighborhood that Formed Shortly After the Big Bang Astrophys. J. Lett. 765 (2013) L12 [astro-ph/1302.3180].

[30] X. Dumusque, et al The Kepler-10 Planetary System Revisited by HARPS-N: A Hot Rocky World and a Solid Neptune-Mass Planet Ap J., 789 (2014) 154 [astro-ph/1405.7881].

[31] Vennes et al, An unusual white dwarf star may be a surviving remnant of a subluminous Type Ia supernova, Science, 357 (2017) 680 [astro-ph/1708.05568].

[32] K. Hattori et al. Old, Metal-Poor Extreme Velocity Stars in the Solar Neighborhood, [astro-ph/1805.03194]

[33] T. Marchetti, E. M. Rossi A.G.A. Brown Gaia DR2 in 6D: Searching for the fastest stars in the Galaxy, [astro-ph/1804.10607].

[34] D.P. Bennett, A. Udalski, I.A. Bond, et al, A Planetary Microlensing Event with an Unusually RED Source Star, [astro-ph/1806.06106].

[35] N.J. Ruffini, A.R. Casey, A hyper-runaway white dwarf in Gaia DR2 as a Type Iax supernova primary remnant candidate [astro-ph/1908.00670].

[36] M. Moniez, Microlensing as a probe of the Galactic structure: 20 years of microlensing optical depth studies, Gen. Rel. Grav. 42, 2047 (2010) [astro-ph/1001.2707]. 
[37] S. I. Blinnikov, Mirror matter and other dark matter models, Phys. Usp. 57 (2014) 183; [ Usp. Fiz. Nauk, 184, (2014) 194].

[38] S. I. Blinnikov, A. D. Dolgov, K. A. Postnov, Antimatter and antistars in the universe and in the Galaxy, Phys. Rev. D 92, (2015) 023516 [astro-ph/1409.5736]

[39] S. Blinnikov, A. Dolgov, N. K. Porayko and K. Postnov, Solving puzzles of GW150914 by primordial black holes, JCAP, 1611, (2016) 036 [astro-ph/1611.00541].

[40] D. Dolgov, S. Porey, Massive primordial black holes in contemporary universe, [astro-ph/1905.10972].

[41] C. Alcock et al. [MACHO Collaboration], The MACHO project: Microlensing results from 5.7 years of LMC observations, Astrophys. J. 542, 281 (2000) [astro-ph/0001272].

[42] J. P. Beaulieu et al., Spectroscopic Studies of the Two Eros Candidate Microlensed Stars, Astron. Astrophys. 299, 168 (1995) [astro-ph/9411051].

[43] P. Tisserand et al. [EROS-2 Collaboration], Limits on the Macho Content of the Galactic Halo from the EROS-2 Survey of the Magellanic Clouds, Astron. Astrophys. 469, 387 (2007) [astro-ph/0607207].

[44] S. Calchi Novati, S. Mirzoyan, P. Jetzer and G. Scarpetta, Microlensing towards the SMC: a new analysis of OGLE and EROS results, Mon. Not. Roy. Astron. Soc. 435, 1582 (2013) [astro-ph/1308.4281].

[45] A. Riffeser, S. Seitz and R. Bender, The M31 microlensing event WeCAPP-GL1/Point-AGAPE-S3: evidence for a MACHO component in the dark halo of M31?, Astrophys. J. 684, 1093 (2008) [astro-ph/0805.0137].

[46] G. Ingrosso, S. Calchi Novati, F. De Paolis, P. Jetzer, A. A. Nucita, G. Scarpetta and F. Strafella, A new analysis of the MEGA M31 microlensing events, Astron. Astrophys. 462, 895 (2007) [astro-ph/0610239].

[47] C.-H. Lee, A. Riffeser, S. Seitz, R. Bender and J. Koppenhoefer, Microlensing events from the 11-year observations of the Wendelstein Calar Alto Pixellensing Project, Astrophys. J. 806, no. 2, 161 (2015) [astro-ph/1504.07246].

[48] S. Mao, Astrophysical Applications of Gravitational Microlensing, Res. Astron. Astrophys. 12, 947 (2012) [astro-ph/1207.3720].

[49] J. Yoo, J. Chaname and A. Gould, The end of the MACHO era: limits on halo dark matter from stellar halo wide binaries, Astrophys. J. 601, 311 (2004) [astro-ph/0307437].

[50] V. Belokurov, N. W. Evans and Y. Le Du, Lightcurve classification in massive variability surveys, Mon. Not. Roy. Astron. Soc. 341, 1373 (2003) [astro-ph/0211121].

[51] V. Belokurov, N. W. Evans and Y. Le Du, Lightcurve classification in massive variability surveys 2: Transients towards the Large Magellanic Cloud, Mon. Not. Roy. Astron. Soc. 352, 233 (2004) [astro-ph/0404232].

[52] K. Griest and C. L. Thomas, Contamination in the MACHO dataset and the puzzle of LMC microlensing, Mon. Not. Roy. Astron. Soc. 359, 464 (2005) [astro-ph/0412443].

[53] N. W. Evans and V. Belokurov, Is there a microlensing puzzle?, [astro-ph/0505167].

[54] D. P. Quinn, M. I. Wilkinson, M. J. Irwin, J. Marshall, A. Koch and V. Belokurov, On the Reported Death of the MACHO Era, Mon. Not. Roy. Astron. Soc. 396, 11 (2009) [astro-ph/0903.1644]. 
[55] M. A. Monroy-Rodriguez and C. Allen, The end of the MACHO era-revisited: new limits on MACHO masses from halo wide binaries, Astrophys. J. 790, no. 2, 159 (2014) [astro-ph/1406.5169].

[56] V. S. Berezinsky, V. I. Dokuchaev and Y. N. Eroshenko, Small-scale clumps of dark matter, Phys. Usp. 57, 1 (2014) [Usp. Fiz. Nauk 184, 3 (2014)] [astro-ph/1405.2204].

[57] F. Ozel, D. Psaltis, R. Narayan and J. E. McClintock, The Black Hole Mass Distribution in the Galaxy, Astrophys. J. 725 (2010) 1918 [astro-ph/1006.2834].

[58] L. Kreidberg, C. D. Bailyn, W. M. Farr and V. Kalogera, Mass Measurements of Black Holes in X-Ray Transients: Is There a Mass Gap, Astrophys. J. 757 (2012) 36 [astro-ph/1205.1805].

[59] K. Postnov and N. Mitichkin, Spins of primordial binary black holes before coalescence, JCAP, 1906 (2019) 044 [astro-ph/1904.00570].

[60] K. Postnov, A. Kuranov and N. Mitichkin, Spins of black holes in coalescing compact binaries, Physics-Uspekhi, 62 (2019) No. 11, [astro-ph/1907.04218].

[61] M. Mirbabayi, A. Gruzinov and J. Noreña, Spin of Primordial Black Holes, [astro-ph/1901.05963].

[62] V. De Luca, et al The initial spin probability distribution of primordial black holes, JCAP 1905 (2019) 018 [astro-ph/903.01179].

[63] W. Zheng et al., A highly magnified candidate for a young galaxy seen when the Universe was 500 Myrs old, Nature, 489 (2012) 406 [astro-ph/1204.2305].

[64] D. Coe et al, CLASH: Three Strongly Lensed Images of a Candidate z 11 Galaxy, Astrophys. J. 762 (2013) 32 [astro-ph/1211.3663].

[65] Chao-Wei Tsai, P.R.M. Eisenhardt et al, The most luminous galaxies discovered by WISE, Astrophys. J. 805 (2015) 90 [astro-ph/1410.1751]

[66] D. Lam et al, Detection of a Lensed $z \approx 11$ Galaxy in the Rest-Optical with Spitzer/IRAC and the Inferred SFR, Stellar Mass, and Physical Size, [astro-ph/1903.08177].

[67] T. Wang et al., A dominant population of optically invisible massive galaxies in the early Universe, Nature, 572 (2019) no.7768, 211 [astro-ph/1908.02372].

[68] T. Hashimoto, et al The onset of star formation 250 million years after the Big Bang, Nature, 557 (2018) 392 [astro-ph/1805.05966].

[69] D. Waters, S. M. Wilkins, T. Di Matteo, Y. Feng, R. Croft and D. Nagai, Monsters in the Dark: Predictions for Luminous Galaxies in the Early Universe from the BlueTides Simulation, Mon. Not. Roy. Astron. Soc. 461, no. 1 (2016) L51 [astro-ph/1604.00413].

[70] F. Melia, The Premature Formation of High-Redshift Galaxies, Astronom. J. 147, no.5 (2014) 120 [astro-ph/1403.0908]].

[71] D.J. Mortlock, et al, A luminous quasar at a redshift of $z=7.085$, Nature, 474 (2011) 616 [astro-ph/1106.6088].

[72] Xue-Bing Wu et al, An ultra-luminous quasar with a twelve-billion-solar-mass black hole at redshift 6.30, Nature 518 (2015) 512 [astro-ph/1502.07418].

[73] M. A. Latif, M. Volonteri and J. H. Wise, Early growth of typical high redshift black holes seeded by direct collapse, Mon. Not. Roy. Astron. Soc. 476, no.4 (2018) 5016 [astro-ph/1801.07685].

[74] Y. Matsuoka et al, Subaru High-z Exploration of Low-luminosity Quasars (SHELLQs). V. Quasar Luminosity Function and Contribution to Cosmic Reionization at $z=6$, Astrophys. J. 869 (2018) 150 [astro-ph/1811.01963]. 
[75] E. Bañados et al An 800-million-solar-mass black hole in a significantly neutral Universe at redshift 7.5, Nature, 553 (2018) 473 [astro-ph/1712.01860]

[76] D.L. Clements et al Dusty Galaxies at the Highest Redshifts Galaxies at High Redshift and Their Evolution Over Cosmic Time, IAU Symposium 319 (2016) 84.

[77] L. Mattsson, The sudden appearance of dust in the early Universe, [astro-ph/1505.04758].

[78] K. K. Knudsen et al, A merger in the dusty, $z=7.5$ galaxy A1689-zD1?, Mon. Not. Roy. Astron. Soc. 466, no.1 (2017) 138 [astro-ph/1603.03222].

[79] B. Venemans et al, Copious Amounts of Dust and Gas in a $z=7.5$ Quasar Host Galaxy, Astrophys. J. Lett. 851 (2017) L8 [astro-ph/1712.01886].

[80] M. Mancini et al, The dust mass in z > 6 normal star forming galaxies Mon. Not. R. Astron. Soc., 451 (2015), 70 [astro-ph1505.01841].

[81] V. Asboth et al, HerMES: A search for high-redshift dusty galaxies in the HerMES Large Mode Survey - Catalogue, number counts and early results, Mon. Not. Roy. Astron. Soc. 462, no. 2 (2016) 1989 [astro-ph/1601.02665]

[82] A. Leśniewska and M.J. Michałowski, Dust production scenarios in galaxies at z 6-8.3, Astron. Astrophys. 624 (2019) L13 [astro-ph/1904.11185].

[83] T. Matsumoto, D. Nakauchi, K. Ioka and T. Nakamura, Jet-powered supernovae of $\sim 10^{5} M_{\odot}$ population III stars are observable by Euclid, WFIRST, WISH, and JWST, Astrophys. J. 823 (2016) 83 [astro-ph/1512.03058].

[84] S. Clesse and J. Garcia-Bellido, Massive Primordial Black Holes from Hybrid Inflation as Dark Matter and the seeds of Galaxies, Phys. Rev. D 92, no.2 (2015) 023524 [astro-ph/1501.07565].

[85] I. Affleck and M. Dine, A New Mechanism for Baryogenesis, Nucl. Phys. B 249 (1985) 361.

\section{DISCUSSION}

BATISTELLI: Do you foresee primordial black holes as a source of spectral distiortion of CMBR?

DOLGOV: Yes, they can create spectral distortion at the level which leads to noticeable constraints in some interesting mass intervals. 